\title{
Two-Dimensional Spatial Resolution in Plane Temperature Monitoring Based on Raman Distributed Temperature Sensor
}

\author{
Junfan $\mathrm{CHEN}^{\mathrm{a}}$, Ning $\mathrm{SUN}^{\mathrm{b}}$ and Zhongxie JIN ${ }^{\mathrm{c}, 1}$ \\ a The Key Laboratory of Optoelectronic Technology \& System, Education Ministry of \\ China, Chongqing 400044, China; chenjf584@outlook.com \\ ${ }^{\mathrm{b}}$ The Key Laboratory of Optoelectronic Technology \& System, Education Ministry of \\ China, Chongqing 400044, China; 413807424@qq.com \\ ${ }^{\mathrm{c}}$ The Key Laboratory of Optoelectronic Technology \& System, Education Ministry of \\ China, Chongqing 400044, China; jinzhongxie@cqu.edu.cn
}

\begin{abstract}
Spatial resolution is an important parameter that characterizes the detection capability of a system, and there are extremely high requirements for spatial resolution in important fields such as the fossil energy industry and nuclear industry. In order to realize the high-precision distributed monitoring of the optical fiber distributed temperature sensing system (DTS), the factors affecting the spatial resolution of the DTS system were analyzed, and a two-dimensional planar temperature field distribution monitoring scheme based on Raman distributed temperature sensor (RDTS) was proposed. In this scheme, based on the layout of the two-dimensional RDTS heat source positioning system, multimode fiber was adopted. After comparing several sensing fiber routing schemes, the $45^{\circ}$ skew $2 \mathrm{D}$ wiring method of sensing fiber was finally selected. According to the experimental results, the spatial resolution of the temperature field distribution in the monitoring area can break through the limitation of the system resolution. It has more application value than the traditional one-dimensional distributed temperature sensing system.
\end{abstract}

Keywords. spatial resolution, two-dimensional positioning

\section{Introduction}

In optics, the ability of the imaging system to separate two objects that are very close together is called the resolution of the optical imaging system. The Abbe imaging principle shows that the optical system creates a Fraunhofer diffraction pattern on the focal plane. Due to the diffraction caused by the limited aperture of the imaging system, the images of two very close points may be inseparable.

In recent years, spatial resolution [1-3] has been involved in more and more fields. The most common are optical, remote sensing satellite radar, image sensors, and medical smart wearable devices represented by artificial skin. Spatial resolution exists in twodimensional plane space [4], which has more research significance and practical use than one-dimensional space.

\footnotetext{
${ }^{1}$ Corresponding Author. Email: jinzhongxie@cqu.edu.cn; Tel.: +86-186-2355-4364
} 
In this paper, we adopt an optimized two-dimensional positioning method based on the traditional RDTS system proposed by Zhang [5] et al., using $45^{\circ}$-inclined-cross-type $\left(45^{\circ}\right.$-ICT) routing rules and supporting algorithms to convert one-dimensional fiber [6] measurement data to two-dimensional planar distribution results. Starting from the networking mode of the RDTS system, the advantages of the networking mode used are introduced, and the factors that affect the spatial resolution of the system are analyzed.

\section{System Configuration and Spatial Resolution}

\subsection{Structure of RDTS system}

The DTS system in this paper was mainly used for real-time monitoring of the heat source in the temperature field [7,8], which needed to meet the two requirements of precise positioning and accurate measurement simultaneously [9]. RDTS system [10-13] is mainly composed of six parts, including transmission module, filter module, photoelectric detection module, DAQ (data acquisition) module, PC (personal computer) and SFL (Sensing Fiber Link). Distributed Fiber temperature measurement system was shown in Fig. 1.

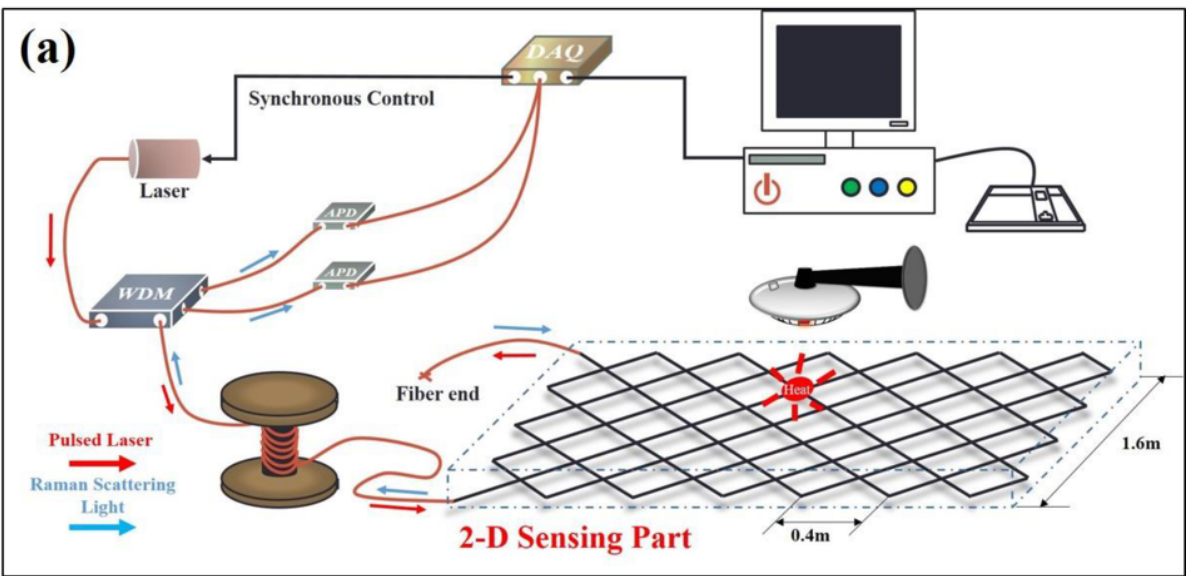

(b)

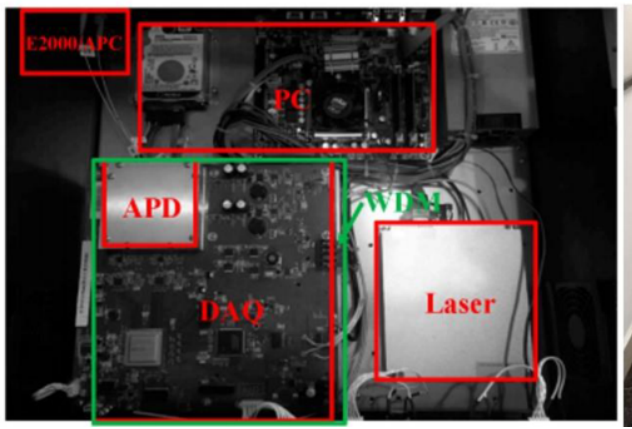

(c)

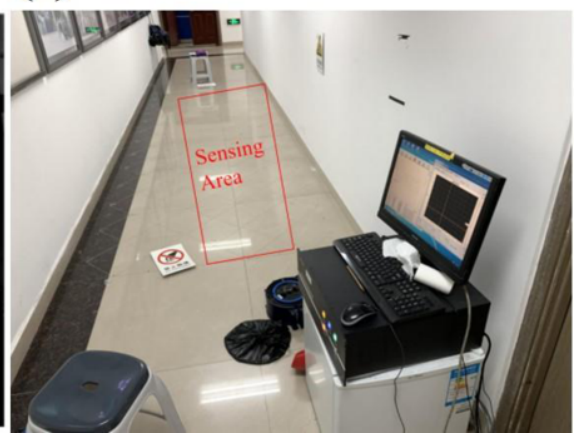

Figure 1. (a) RDTS-Based Two-Dimensional temperature field distribution monitoring;

(b)(c) Experimental platform 
In this experiment, we used a high-power pulsed laser with a wavelength of 1550 $\mathrm{nm}$, a pulse width of $7 \mathrm{~ns}$, and a frequency of $8 \mathrm{kHz}$ as the light source. The pulse is transmitted into the SFL through wavelength division multiplexing (WDM), using two high-sensitivity, low-noise avalanche photodiodes (APDs) with a bandwidth of $150 \mathrm{MHz}$ to simultaneously detect the weak backscattered light along the SFL. Use a 12-bit 250 $\mathrm{MHz}$ sampling frequency data acquisition (DAQ) card for signal processing, and transfer the processed results to a PC for graphic display. The fiber end is bent with a small radius to avoid reflection effects.

\subsection{Spatial Resolution of RTDS}

In a two-dimensional plane temperature distribution field, if two heat sources can be distinguished by the distributed optical fiber temperature sensing system, the minimum distance between the two heat sources is called the spatial resolution. In fact, in the distributed optical fiber temperature sensing system, there is another parameter that characterizes the resolution ability: System resolution [11,14], but the difference is, the spatial resolution is more focused on the diameter between any two temperature regions on a two-dimensional plane. The system resolution can only quantitatively indicate the one-dimensional minimum temperature resolution distance. The spatial resolution is more suitable for measuring the two-dimensional heat source discrimination accuracy of the distributed optical fiber sensing system on a two-dimensional plane.

\section{Experiment}

\subsection{Fiber Distribution}

The traditional distributed optical fiber temperature sensing system had a linear type and an $\mathrm{S}$ type. The linear type is suitable for scenarios where only one-dimensional spatial temperature distribution data is required, so the coordinates of the heat source in one dimension can only be determined, and the two-dimensional spatial temperature distribution cannot be obtained, and the scope of application is very narrow. The S-type sensor fiber distribution was as Fig.2(a):

(a)

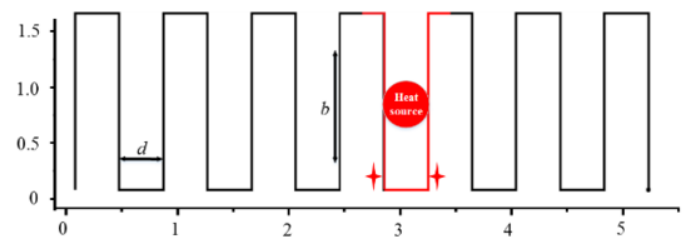

(b)

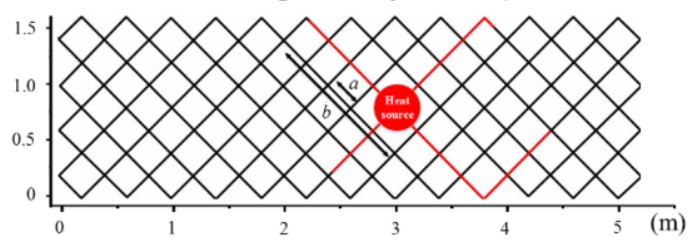

Figure 2. (a) S-type distributed sensor fiber networking; (b) $45^{\circ}$ diagonally distributed sensor fiber networking [5] 
We have introduced a new two-dimensional matrix positioning method to modify the algorithm without modifying the materials or hardware, which can reduce the cost of the RDTS system. The specific distribution is shown in Fig. 2(b). The minimum unit side length was $a=d / 1.414$, and the red fiber segment corresponded to the high temperature area of the sensor fiber link. A sufficiently long sensor fiber link was distributed across the two-dimensional plane, dividing the two-dimensional plane into many Uniform and independent grid. According to the $45^{\circ}$ diagonal span distribution we used, we kept the slope of the sensing fiber link to the two-dimensional plane coordinate system at 1 or -1 . When the sensing fiber link reaches the edge of the sensing area, the slope becomes the opposite of the original. The corner of the grid, which was the intersection of the sensing fiber link, carried the temperature distribution information on the sensing fiber and was used to define the position in space. This method could significantly reduce crosstalk, because any two adjacent intersections contained at least a pair of fiber segments located far apart. And we can obtain the same positioning accuracy in both directions. Compared with the linear distribution, this fiber network can greatly increase the coverage of the optical fiber to the area to be tested, reduce the probability of missed.

The representation of the matrix is shown in Fig. 2(b). The plane was divided into many grids by optical fibers. Each vertex of the grid records the temperature information of the sensing fiber link, which is used for spatial positioning. The matrix corresponds to the intersections, the row and column of the matrix correspond to the $2 \mathrm{D}$ coordinates of the vertices, and the value of every element in the matrix corresponds to the temperature of the intersection point [5].

We conducted multiple tests on different locations in the experiment to verify the accuracy and effectiveness of the fiber networking. We can get that the positioning accuracy of this method is half of the diagonal of the grid $(d / 2)$.

(a)

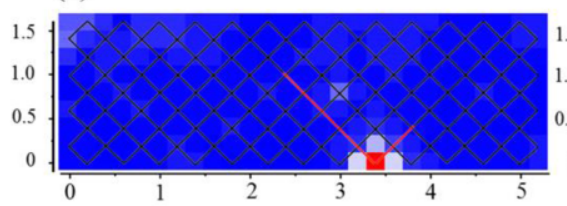

(c)

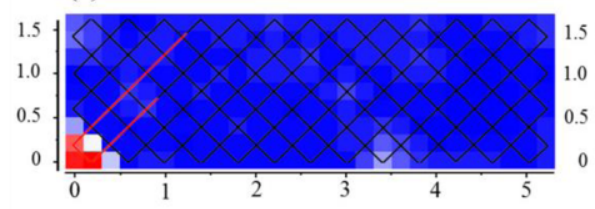

(b)

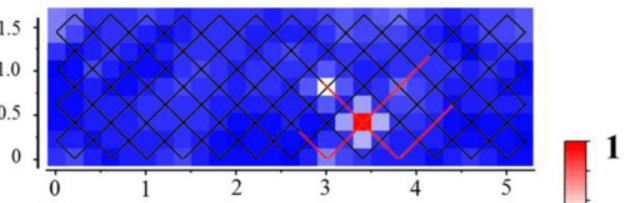

(d)

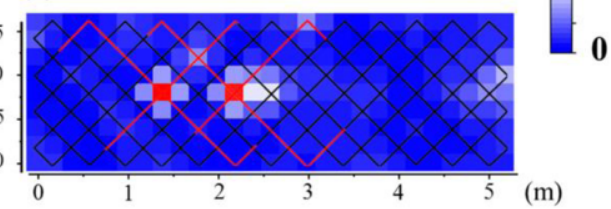

Figure 3. Temperature detection at different intersections [5]

As shown in Fig.3(a), the inflection point of the edge or corner was regarded as the intersection point. In Fig.3(b), when the heat source was located near the edge, there were high-temperature inflection points and intersection points. After setting the intersection priority to be higher than the inflection point priority, determined that the actual heat source was near the intersection. We also tested the situation of two heat sources close together, as shown in Fig.3(d). These two heat sources were difficult to distinguish because there were four intersection points of abnormal temperature. In the matrix, an additional priority was set according to the position of the crossing point of the abnormal temperature optical fiber segment (The priority would be higher if the 
intersection is at the center of the high-temperature fiber segment instead of the edge position).

\subsection{Determination of Spatial Resolution}

The measurement of spatial resolution, that is, the measurement of the minimum distance of two heat sources which can be separated, requires the existence of a saddle point (minimum point) shown in Fig.4. Since our experimental system measures the temperature once at $0.4 \mathrm{~m}$, and the grid side length is $0.28 \mathrm{~m}$. When the distance between two heat sources is shorter than $0.28 \mathrm{~m}$, they cannot be separated. So at this time we need a saddle point, that is, the existence of a low temperature point.

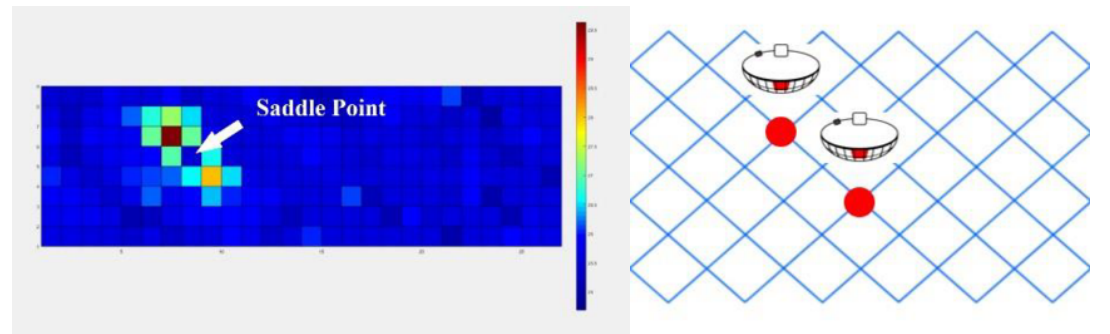

Figure 4. Temperature distribution graph displayed in MATLAB

There was an obvious low-temperature grid between the two heat sources. This is because, under the linear interpolation algorithm, when there is only one high temperature point at the intersection, the grid cannot be displayed in a high temperature state. Because the temperature within $0.4 \mathrm{~m}$ was displayed the same, the temperature of the same fiber where the two heat sources were located showed high temperature, while the other fiber at the cross point showed normal temperature. In this way, it can be judged that there were two heat sources.

We regarded the three heat sources as one heat source, and the gap between the three heat sources was far less than $0.4 \mathrm{~m}$, so it can be ignored. At this time, after being calculated by the program, it was displayed in MATLAB as Fig.5

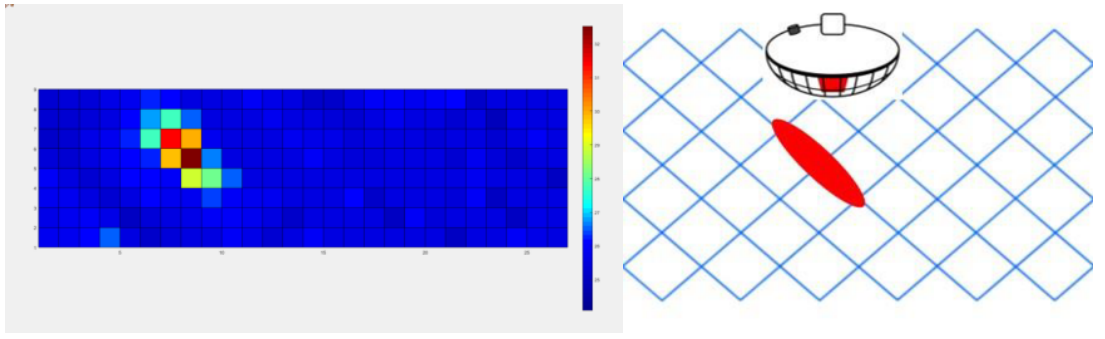

Figure 5. Temperature distribution graph of 3 connected heat sources

At this time, we can see that the temperature of the three grids has become higher together. There was no obvious low-temperature grid inside the heat source. We can judge that this heat source is just one heat source instead of two heat sources separated by a certain distance. According to the above analysis, at the intersection, the spatial resolution was $0.28 \mathrm{~m}$, that is, the distance between the two heat sources at the 
intersection was at least $0.28 \mathrm{~m}$ to be distinguished. The same conclusion can be drawn after repeated experiments.

\subsection{Factors Affecting the Spatial Resolution of the System}

3.3.1. Resolution of RDTS System. The resolution of the RDTS system greatly affected the spatial resolution. It refers to the minimun length that the system can distinguish by measuring the temperature field along the one-dimensional length of the fiber. It is mainly determined by the pulse width $\Delta t$, photodetector response time $\tau$, and analogto-digital conversion time $T_{A / D}$, which are expressed as Eq.(1) $[15,16]$ :

$$
\begin{aligned}
\Delta L_{1} & =\frac{v \Delta t}{2} \\
\Delta L_{2} & =\frac{v \tau}{2} \\
\Delta L_{3} & =\frac{v t_{A / D}}{2}
\end{aligned}
$$

$v$ is the propagation speed of light wave in optical fiber. The actual resolution of the RDTS system depends on the largest of the three:

$$
\Delta L=\max \left\{\Delta \mathrm{L}_{1}, \Delta \mathrm{L}_{2}, \Delta \mathrm{L}_{3}\right\}
$$

The system resolution of the system used in this experiment was $0.4 \mathrm{~m}$, so when the area of the heat source used was smaller than $0.4 \mathrm{~m} \times 0.4 \mathrm{~m}$, the temperature distribution diagram showed the difference between the temperature profiles of the two heat sources. Because the system resolution determined the cumulative average interval of the RDTS, it also determined the minimum area where the temperature can be detected, which in turn determined the spatial resolution in the two-dimensional plane.

3.3.2. Distribution Density. The networking density of the RDTS system can make up for the limitations caused by spatial resolution. We doubled the distribution density of optical fiber, as shown on Fig.6.

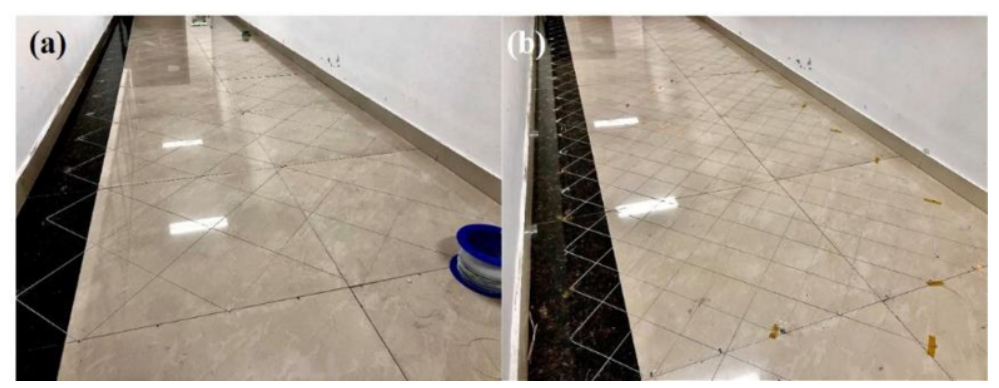

Figure 6. (a)Original distribution density of fiber; (b)Double distribution density of fiber

The heat source is changed from covering one intersection point to now covering two intersection points. There was a section of fiber with no high temperature alarm 
between the intersection points as the reference fiber. The temperature diagram in MATLAB was shown in Fig.7:

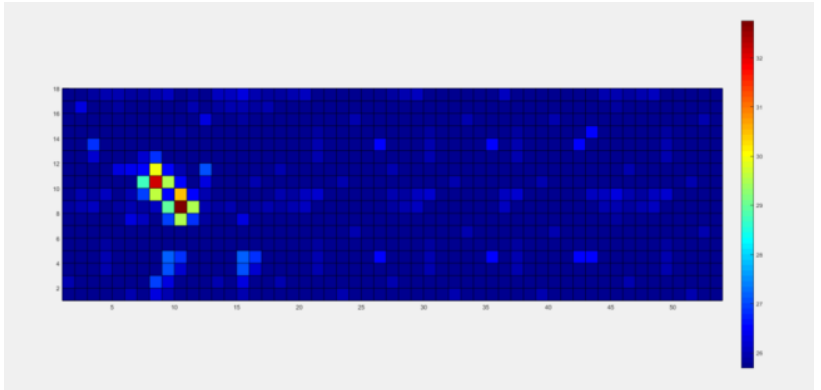

Figure 7. Temperature distribution graph of multiple heat sources in double-density distribution of optical fiber

There was a clear line of low-temperature optical fiber boundary between the two heat sources, and it was judged as two heat sources at this time. We can know that if the two heat sources were placed under the single-density fiber distribution, they cannot be separated because they are in the same temperature measurement interval, but they can be separated in the case of double density. We can be sure that density did affect spatial resolution.

For one heat source, it can be seen that the high temperature area was a connected heat source in Fig.8, which was consistent with the actual situation.

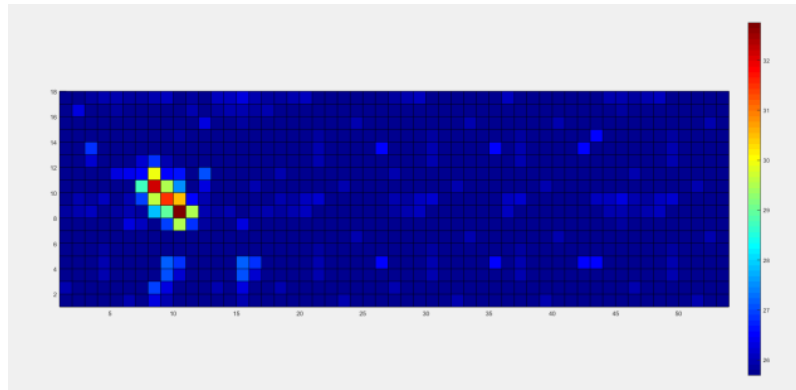

Figure 8. Temperature distribution graph of one heat source in double-density distribution of optical fiber

At this time, the spatial resolution of the system was $0.14 \mathrm{~m}$, that is, at least $0.14 \mathrm{~m}$ between the two heat sources can they be distinguished. Therefore, it can be seen that the spatial resolution of the RDTS system is related to the laying density of the optical fiber, which can make up for the limitation caused by the spatial resolution. The denser the optical fiber is laid, the more judgment areas are available, and the higher the spatial resolution is. If the optical fiber is doubled in density, the spatial resolution will be doubled.

\section{Conclusion}

This paper explained the spatial resolution of the RDTS system and gave the structure of the RDTS system. The $45^{\circ}$ diagonal span fiber laying method is used to derive a method 
for converting one-dimensional sensing data into planar two-dimensional temperature field distribution data. Use MATLAB to calculate the temperature distribution, and give the block diagram and content. The spatial resolution of the RDTS system is related to the system resolution and fiber distribution density. The two-dimensional distribution of optical fibers greatly improves the spatial resolution. Through a higher distribution density of optical fibers and a DTS with $0.4 \mathrm{~m}$ system resolution, we obtained a spatial resolution of $0.14 \mathrm{~m}$. Higher spatial resolution is vital to the practical application of the technology.

\section{References}

[1] M.A. Soto, T. Nannipieri, A. Signorini, A. Lazzeri, F. Baronti, R. Roncella, G. Bolognini, F. Di Pasquale, Raman-based distributed temperature sensor with $1 \mathrm{~m}$ spatial resolution over $26 \mathrm{~km}$ SMF using lowrepetition-rate cyclic pulse coding, Opt. Lett. 36 (2011), 2557-2559.

[2] A.K. Sang, M.E. Froggatt, D.K. Gifford, S.T. Kreger, B.D. Dickerson, One Centimeter Spatial Resolution Temperature Measurements in a Nuclear Reactor Using Rayleigh Scatter in Optical Fiber, IEEE Sens. J. 8 (2008), 1375-1380.

[3] S.D. Dyer, M.G. Tanner, B. Baek, R.H. Hadfield, S.W. Nam, Analysis of a distributed fiber-optic temperature sensor using single-photon detectors, Opt. Express 20 (2012), 3456-3466.

[4] M. Sun, Y.Q. Tang, S. Yang, J. Li, M.W. Sigrist, F.Z. Dong, Fire Source Localization Based on Distributed Temperature Sensing by a Dual-Line Optical Fiber System, Sensors 16 (2016), 829.

[5] C. Zhang, Z.X. Jin, RDTS-Based Two-Dimensional Temperature Monitoring with High Positioning Accuracy Using Grid Distribution, Sensors 19 (2019), 4993.

[6] A.H. Hartog, A.P. Leach, M.P. Gold, Distributed temperature sensing in solid-core fibres, Electron. Lett. 21 (1985), 1061-1062.

[7] J. Zhang, P. Wei, Q. Liu, Monitoring a Heatsink Temperature Field Using Raman-Based Distributed Temperature Sensor in a Vacuum and $-173^{\circ} \mathrm{C}$ Environment, Sensors 19 (2019), 4186.

[8] A.J. Rogers, Polarisation optical time domain reflectometry, Electron. Lett. 16 (2007), 489-490.

[9] K.L. Wei, Z.Y. Wen, J. Zou, et al, Based on OTDR prospective distributed optical fiber temperature alarm system, Piezoelectrics \& Acoustooptics 32 (2010), 329-332.

[10] I. Toccafondo, T. Nannipieri, A. Signorini, E. Guillermain, J. Kuhnhenn, M. Brugger, F.D. Pasquale, Raman Distributed Temperature Sensing at CERN, IEEE Photonic Tech. Lett. 27 (2015), 2182-2185.

[11] I. Laarossi, M.Á. Quintela-Incera, J.M. López-Higuera, Comparative Experimental Study of a HighTemperature Raman-Based Distributed Optical Fiber Sensor with Different Special Fibers, Sensors 19 (2019), 574.

[12] B. Yan; J. Li, M. Zhang, J. Zhang, L. Qiao, T. Wang, Raman Distributed Temperature Sensor with Optical Dynamic Difference Compensation and Visual Localization Technology for Tunnel Fire Detection, Sensors 19 (2019), 2320.

[13] Z. Wang, X.H. Sun, Q. Xue, et al, an optical fiber-folded distributed temperature sensor based on Raman backscattering, Optics \& Laser Technology 93 (2017), 224-227.

[14] B.N. Sun, J. Chang, J. Lian, et al, Accuracy improvement of Raman distributed temperature sensors based on eliminating Rayleigh noise impact, Opt. Commun 306 (2013), 117-120

[15] F. Ning, Y. Zhu, H.J. Cui, X.Q. Li, Z.X. Jin, A Linear Correcting Algorithm for Improving Space Resolution of Distributed Optical Fiber Raman Temperature Measurement System, Acta Photonica Sinica 4 (2012), 408-413.

[16] X.F. Shi, Z. Li, Z.Q. Cai, Optical fiber distributed temperature measurement system and precision analysis, Measurement and Control Technology 21 (2002), 9-12. 